\title{
Pure large cell neuroendocrine carcinoma of the gallbladder, is surgical relentlessness beneficial? A case report and literature review
}

\author{
Anisse Tidjane ${ }^{1}\left(\mathbb{D} \cdot\right.$ Nabil Boudjenan $^{1} \cdot$ Aicha Bengueddach $^{2} \cdot$ Amin Kadri $^{3} \cdot$ Nacim Ikhlef $^{1}$. \\ Noureddine Benmaarouf ${ }^{1} \cdot$ Benali Tabeti $^{1}$
}

Received: 16 June 2020 / Accepted: 17 November 2020 / Published online: 3 January 2021

(c) The Japan Society of Clinical Oncology 2021

\begin{abstract}
Pure large cell neuroendocrine carcinoma of the gallbladder is a rare disease. However, the prognosis of this aggressive tumor is poor with short survival after diagnosis. We are describing in this manuscript a case of pure large cell neuroendocrine carcinoma in which survival exceeded 26 months, after performing two curative surgeries. We are reporting the case of a 68-year-old woman with a history of recovered right breast carcinoma and operated 6 years later for a completely asymptomatic gallbladder tumor of $31 \mathrm{~mm}$. In this case, curative surgery was performed allowing monobloc resection of the gallbladder and the hepatic segments IVb and $\mathrm{V}$, a lymph node dissection was performed to. The histological examination of the specimen and immunohistochemistry confirms that the tumor was a grade 3 pure large cell neuroendocrine carcinoma of gallbladder with lymph node invasion, the hepatic and biliary surgical margins were free. Postoperative adjuvant chemotherapy was administered and the evolution was eventless until the discovery at 20 months of a lymph node considered being metastatic recurrence. A second surgery was performed allowing removal of three lymph nodes. This time, a different protocol of chemotherapy was administered to our patient who remains alive and without recurrence at 26 months from her first surgery. Surgical relentlessness with free margins resections associated with appropriate chemotherapy probably improves the survival of patients suffering from this rare and aggressive tumor.
\end{abstract}

Keywords Hepatobiliary $\cdot$ Gallbladder $\cdot$ Large cell $\cdot$ Neuroendocrine $\cdot$ Outcomes $\cdot$ North-africa

\section{Introduction}

Neuroendocrine carcinoma tumors of the gallbladder (GBNEC) is a rare disease, which counts for less than $2 \%$ of all gallbladder neoplasms, and represent less than $0,5 \%$ of all neuroendocrine carcinoma tumors (NEC) localizations.

Anisse Tidjane

tidjane.anisse@univ-oran1.dz

Nabil Boudjenan

nabprof@hotmail.com

Aicha Bengueddach

bengueddach@live.fr

Amin Kadri

kadridiagnostic@gmail.com

Nacim Ikhlef

Ikhlef2000@yahoo.fr

Noureddine Benmaarouf

Benmaarouf_noureddine@hotmail.com
NEC are divided into three groups, small cell NEC, large cell NEC, and mixed adenocarcinoma-neuroendocrine tumor [1].

Usually, the diagnosis of this cancer is commonly made accidentally next to the gallbladder histological examination after performing a cholecystectomy. In some cases, when the

Benali Tabeti

btabeti@yahoo.fr

1 Department of Hepatobiliary Surgery and Liver Transplantation, EHU-1st November 1954, Department of Medicine, University of Oran 1, B.P 1510 El Menaouer, 31000 Oran, Algeria

2 Department of Oncology, EHU-1st November 1954, Department of Medicine, University of Oran 1, Oran, Algeria

3 Laboratoire d'Anatomie Pathologique Dr. Kadri, Oran, Algeria 
gallbladder tumor is detected at radiological examinations, the diagnosis is confirmed at histological examination of biopsy or surgical specimen. Pure large cell GBNEC is a rare neoplastic disease, only a few cases are reported in the literature [2]. A recent publication suggests the relationship between the history of radiotherapy and the occurrence of grade 3 neuroendocrine tumors at irradiated sites and organs [3].

We report the case of pure large cell GBNEC grade 3 occurred 6 years after radiotherapy for right breast adenocarcinoma. In whom, after performing two consecutive surgeries both for curative purposes with free margins specimens, we obtained a survival greater than 26 months.

\section{Case report}

A 68-year-old female patient with no family history of cancer and with a personal history of ductal cancer of the right breast and type 2 diabetes. For her breast cancer, the patient had received treatment 6 years before; at the time a conservative surgical treatment of this tumor located in the lower outer quadrant of the right breast followed by radiotherapy (65gray), chemotherapy and hormonotherapy was performed. Then, during the planned follow-up, a gallbladder tumor was discovered on routine abdominal ultrasonography.

At the time of admission in our department specialized in hepatobiliary and pancreatic surgery, the patient was asymptomatic in a good general condition, without weight loss, $\mathrm{BMI}=31$. The clinical examination of the abdomen was normal. An abdominal MRI was performed and showed a tumor in the fundus of the gallbladder, visible in low signal intensity in the $\mathrm{T} 2$ sequence and high signal intensity in diffusion sequence, with intense contrast enhancement after injection of Gadolinium. Moreover, three metastatic lymphadenopathies were found in the hepato-gastric ligament sized respectively $18 \mathrm{~mm}, 16 \mathrm{~mm}$, and $27 \mathrm{~mm}$. However, there was no radiologic evidence of liver metastases or peritoneal carcinomatosis (Fig. 1). The thoracic- abdominal- pelvic CT-scan did not show any other suspect founds. Blood tests were featureless and showed a normal rate of CA $19-9=15,78 \mathrm{u} / \mathrm{ml}$ and $\mathrm{CEA}=1,32 \mathrm{ng} / \mathrm{ml}$,

After discussion in the multidisciplinary committee, the patient was operated, surgical exploration showed the presence of a tumor involving the fundus of the gallbladder with evidence of hepatic invasion, and adenomegaly at the hepatoduodenal ligament, no evidence of carcinomatosis, the intraoperative ultrasonography confirmed that the liver was free from any metastasis. So a lymphadenectomy of the hepatoduodenal ligament was performed and extended to retro-pancreatic and coeliac lymph nodes, in association with monobloc resection of the gallbladder and the segment $4 \mathrm{~b}$ and 5 of the liver (Fig. 2). The postoperative period was eventless and the patient was discharged on the seventh postoperative day.

Macroscopical examination of the specimen confirmed the presence of a vegetative white hemorrhagic tumor involving the fundus and the body of gallbladder measuring (30 $\mathrm{mm} \times 25 \mathrm{~mm} \times 14 \mathrm{~mm}$ ).

Histopathology shows a neuroendocrine morphology such as organoid nesting, trabecular growth, rosette-like structure, and areas of a solid nest forming cribriform patterns with perineural invasion and numerous vascular invasion. The tumor cells were large with high mitotic activity $>50 / 2 \mathrm{~mm}^{2}$, involving all layers of the gallbladder, with extensions to the liver and peritoneum, where the liver and cystic resection margins were clear.

The product of the lymph node dissection had brought back five nodes of which four were invaded, the tumor was classified P T3 N2 M0 according to the classification of the AJCC "8th edition", immunohistochemical staining shows diffusely and strongly positivity of Chromagranine A, synaptophysin, CD56, and Ki-67 at 80\% (Figs. 3a, b, c).

Based on a discussion that was carried out in the multidisciplinary committee of the west Algerian network of neuroendocrine tumors, the patient had received standard
Fig. 1 MRI examination, white arrow: tumor of the gallbladder with hepatic invasion. red arrow: invaded lymphadenopathy
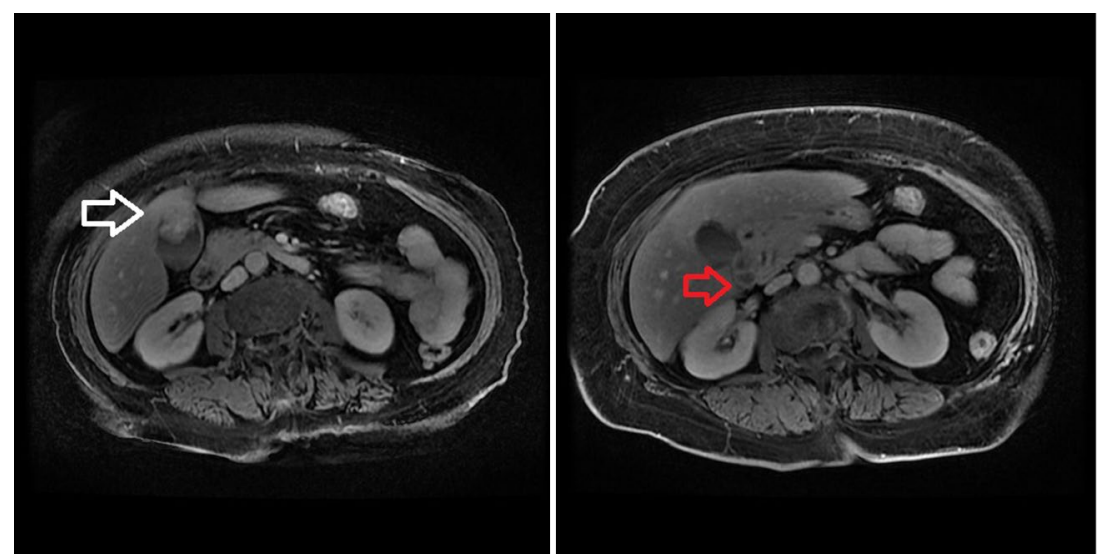


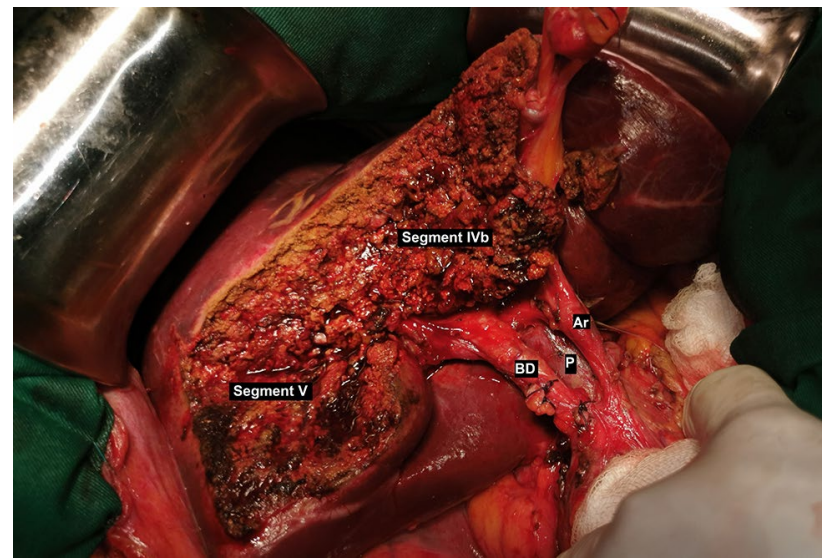

Fig. 2 Perioperative view: bisegmentectomy $\mathrm{Vb}$ and $\mathrm{V}$ removing the tumor and the gallbladder bed in one piece, with lymph node dissection, individualization and exposure of the main bile duct (BD), the portal vein $(\mathrm{P})$ and the hepatic artery $(\mathrm{Ar})$ adjuvant chemotherapy (Etoposide -Cisplatin) four cycles, she was monitored every 3 months during the first year by performing a CT-scan, then every 6 months for the following next year.

Twenty months after surgery, the patient developed a suspected recurrence of a disease with the appearance of a large left lateral para-aortic lymphadenopathy of $51 \mathrm{~mm}$ on control imaging (Fig. 4). Unfortunately, the 18 FDG-PETCT was unavailable; therefore, it could not be realized. After discussion in the multidisciplinary committee, a second surgery was performed allowing the resection of the detected metastatic lymph node and to perform an additional lymphadenectomy of the left lateral-aortic chain having allowed the dissection of two other lymph nodes (Fig. 5). The histological examination had confirmed that all removed lymph nodes were invaded by the same histological type of tumor previously described with capsular rupture (Figs. 6, 7). The patient received four cycles of (5-Fluoro-uracil + Oxaliplatin) and remains alive without any disease recurrence on control CT-scan at 26 months from her first surgery.
Fig. 3 a Gallbladder parietal involvement of muscular layer by pure large cells neuroendocrine carcinoma $(10 \times$, coloration: H\&E). b lymph node involvement by large cells neuroendocrine carcinoma $(4 \times$ coloration: H\&E). c Microscopic histological appearance after immunohistochemical stains $(4 \times)$. 1- Microscopic view at $H \& E$ coloration $(4 \times)$. 2- Immunohistochemical staining showing that the large cells were positive for chromogranin. 3- Immunohistochemical staining showing that large cells were positive for synaptophysin. 4- Cancer cells showed a Ki-67 index of $80 \%$
A

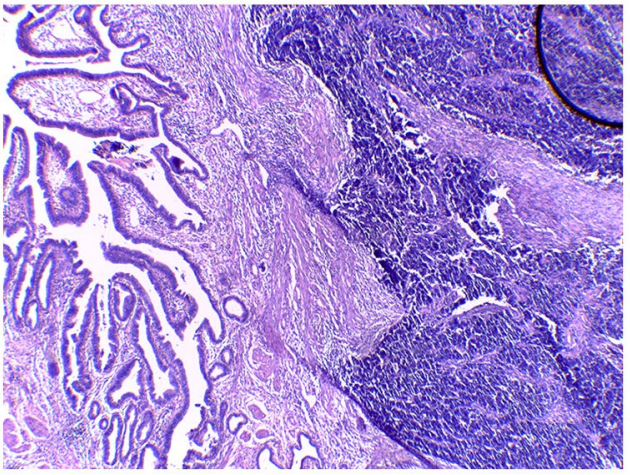

B

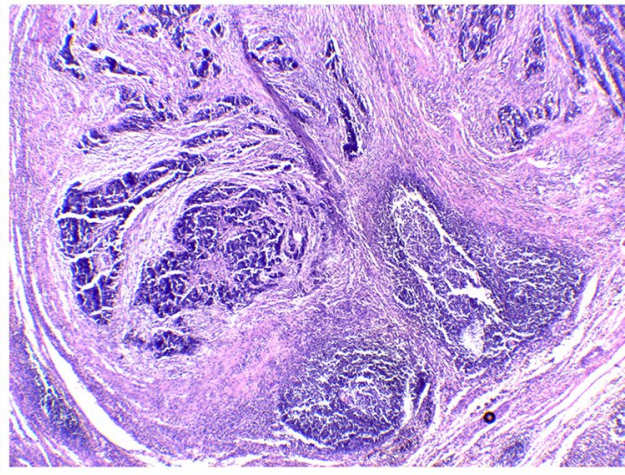

\section{C}
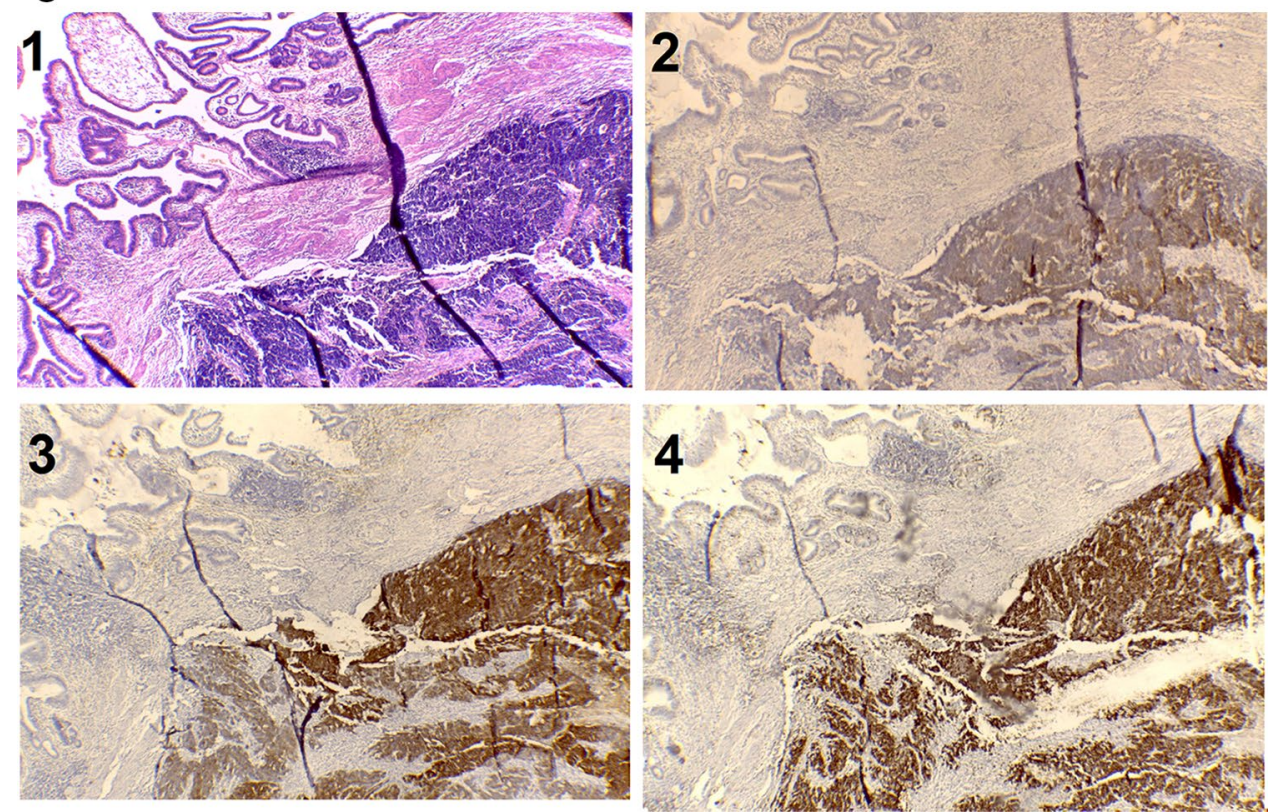


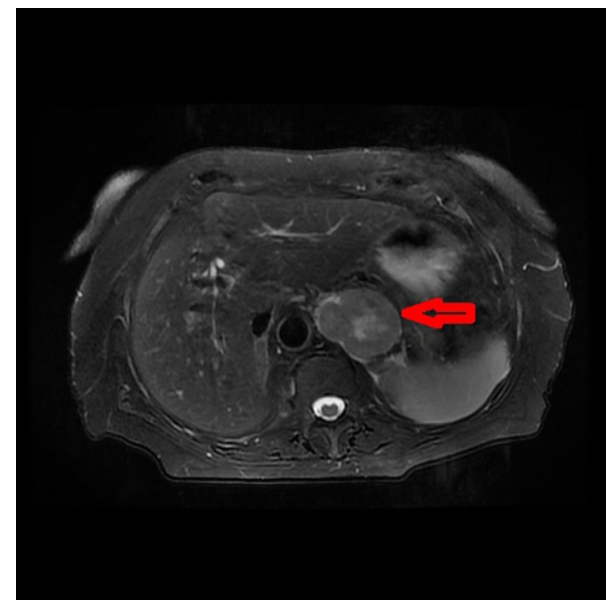

Fig. 4 Left lateral para-aortic lymph node of $51 \mathrm{~mm}$ on MRI

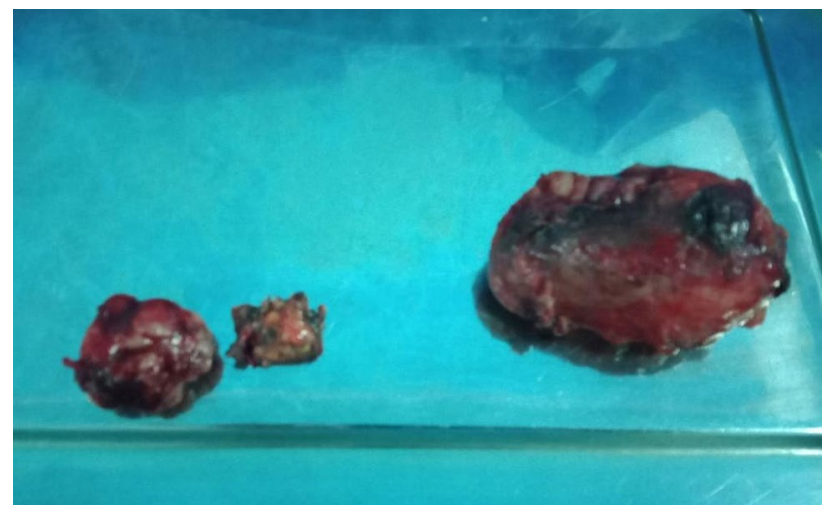

Fig. 5 Product of lymph node dissection: three lymph nodes of different sizes

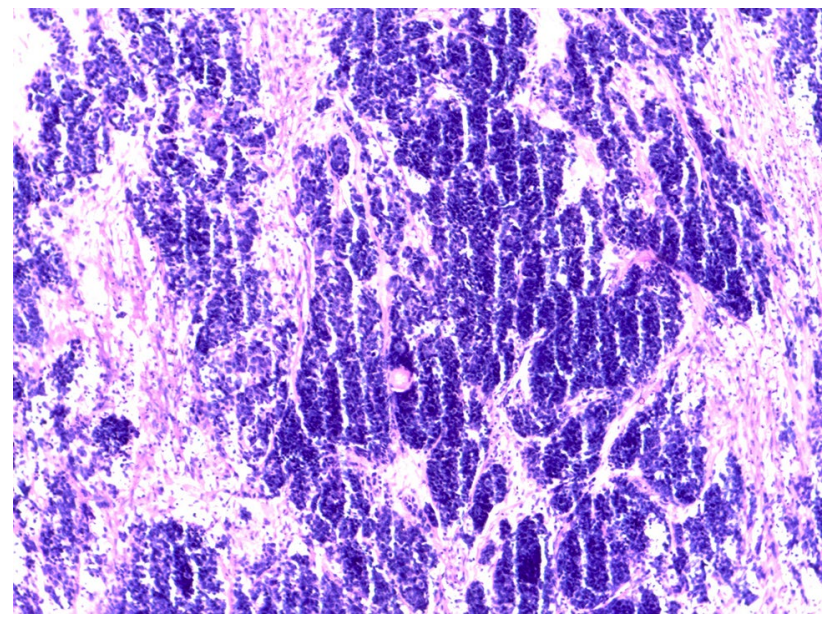

Fig. 6 Lymph node involvement by neoplastic cells showing a sheetlike growth with trabecular and rosette-like patterns (H\&E 20X)

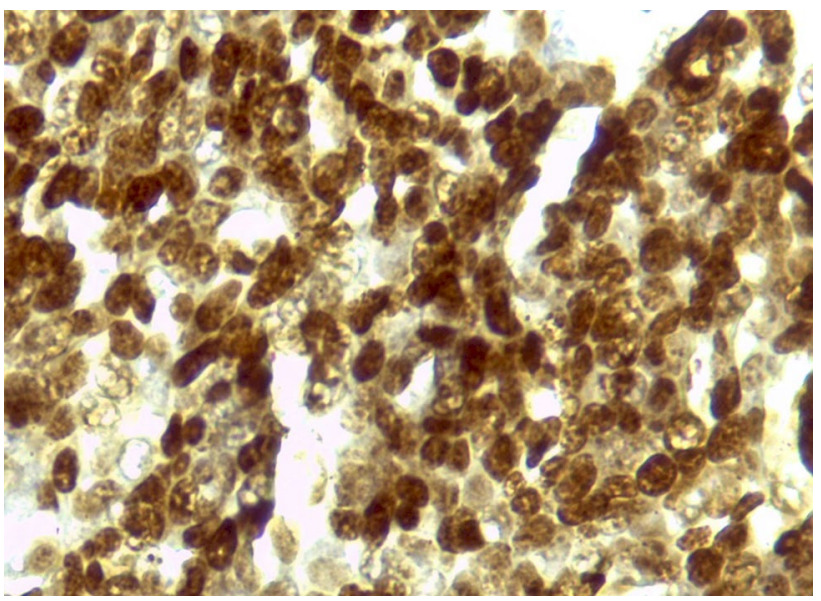

Fig. 7 Cells showed a Ki-67 index of 90\% (40X)

\section{Discussion}

In Algeria, gallbladder cancer represents $4 \%$ of women's incident cancers [4], Primary GBNEC are rare and represent $2,3 \%$ of all gallbladder neoplasm and less than $0,5 \%$ of all gastrointestinal NEC [5].

The first case of pure large cell GBNEC was described in 2000 by Papotti et al. [6], since only 14 pure large cell GBNEC was described in the literature, our case is the 15th, and the first African reported case.

The liver is considered as an organ at risk during irradiation of right breast cancer [7], a recent publication suggests the presence of a relationship between the history of radiotherapy and the occurrence of grade $3 \mathrm{NEC}$ at irradiated sites and organs [3]. In our case, the GBNEC was diagnosed 6 years after radiotherapy for right breast cancer.

Symptoms described by patients with GBNEC are nonspecific, often related to bile duct tension such as biliary colic or acute cholecystitis or jaundice in rare cases [8]. Mostly, this disease is discovered after performing cholecystectomy for cholecystitis or cholelithiasis, in some cases, the tumor is detected on imaging leading to biopsy or surgical resection $[6,9]$. The radiologic patterns of GBNEC and gallbladder adenocarcinoma are similar. However, Kim et al. conclude that the well-defined margin associated to larger hepatic and lymph nodes metastases are useful CTscan differentiators of GBNECs from adenocarcinoma [10]. In all cases, the histopathology confirms the unsuspected diagnosis of GBNEC $[8,9]$. In our case, the diagnosis of a gallbladder tumor was made during the periodic follow-up of a cured right breast cancer in an asymptomatic woman. The abdominal ultrasonography revealed the gallbladder tumor and the MRI that was performed before the surgery had revealed lymphadenopathy and had predicted the absence of liver metastases, as well, the neuroendocrine nature of the tumor was not suspected. The 18FDG-PET-CT can be 
used to confirm the GBNET. However, it is not practical, to use this expensive imagery and rarely available in all cases of suspected gallbladder cancer when histopathology allows having the diagnosis on micro-biopsy or cytology guided by standard imagery. On the other hand, this imaging is useful in the search for an undetected primary tumor and the search for metastases [11]. In our case, the R0 surgical resection was proposed without prior biopsy to avoid dissemination, this decision was made by the multidisciplinary committee of EHU-1st November 1954 Hospital.

To confirm the diagnosis of NEC on histological examination, immunohistochemistry is essential as these tumors are positive for chromogranin A, synaptophysin, CD56 and Ki67 [12].

In our case, radical resection surgery of the gallbladder tumor associated to liver segments $4 \mathrm{~b}$ and 5 resection and lymph nodes dissection was performed, the hepatic and biliary surgical margins of the resection specimen were not involved and lymph nodes showed tumoral invasion on final histological examination. This radical surgical solution is associated with a better overall survivor in the case of an adénocarcinoma of the gallbladder [13]. However, the benefits of this surgery were not studied in cases of GBNEC. Besides, radical surgery provides sufficient hepatic free margins with R0 resection and more precise staging of the disease because of lymph node dissection, hence, leading to better evaluation for the prescription of adjuvant therapy. According to Ayabe et al. surgical resection with negative margins is associated with better overall survival after surgery of the GBNEC, others poor prognostic factors were: large cell NECs, the tumor size larger than $25 \mathrm{~mm}$, AJCC stage and tumors grade 3 (5). Moreover, it appeared that the GBNEC have a worse prognosis compared to other gastrointestinal NECs with poor overall survival. Thus, our patient cumulate several bad prognosis factors, the only favorable factor was the surgical resection with negative margins. After curative resection, adjuvant chemotherapy type Etoposide + cisplatin in four cycles is recommended with a monitoring schedule to detect any recurrence of disease [12].

In case of recurrence, rare are the publications that plead in favor of relentless surgery, in our case after lymph node recurrence at 20 months, the decision to propose a second surgery was validated by the Multidisciplinary Committee and accepted by the patient. This second surgery allowed resection of all metastatic lymphadenopathy. In our case, given the failure of the adjuvant chemotherapy administered after the first curative surgery, a change of protocol was decided with the administration of cytotoxic drugs based on Oxaliplatin, this attitude is recommended by some authors for grade 3 digestive NECs tumors that do not respond well to the Cisplatin Etoposide protocol [12, 14-16]. The chemotherapy used in patients affected by GBNEC is inspired by treatments administered to other digestive NECs, and without any conviction that the results of these treatments are efficient on biliary NEC patients, in any case, it also appears that large cell GBNECs have a poor response to chemotherapy [5, 17].

Abutaka et al. had reported a case of pure large cell GBNEC where relentless surgery after a first $\mathrm{R} 0$ resection of an NEC of the gallbladder, followed by two hepatic metastasectomy with lymph node dissection had prolonged survival to 26 months [18].

Shimano et al. reported a case of GBNEC, in which, the tumor was not surgically extirpable. The down-staging was obtained by radio-chemotherapy, 50 Gy radiotherapy associated with intra-arterial chemotherapy, the patient had undergone a right portal embolization before an extended right trisegmentectomy, then surgery with radiotherapy was performed for metachronous brain metastases, the patient has finally survived for 69 months [19].

Shapera et al. obtained a survival of 19 without recurrence after performing a radical cholecystectomy with lymph node dissection followed by chemotherapy in a patient where the tumor was discovered after performing a laparoscopic cholecystectomy [20]. Other authors had obtained appreciable survival after carcinological resection associated with lymph node dissection with no recurrence of this aggressive malignant rare pathology [21].

Through these three cases, no particular conclusion can be made. However, it is assumed that the reoperation is beneficial with prolonged survival whenever a curative resection with free margins is performed even for cancer recurrence. Whatever, all decisions have to be discussed and validated by the multidisciplinary committee.

A table summarizes a literature review of published cases of a pure large cell GBNEC with a summary of proposed treatments and results (Table 1).

The pure large cell neuroendocrine carcinoma of the gallbladder is a rare and aggressive tumor, with a short survival compared to other neuroendocrine tumors of the digestive tract. Performing radical excision surgery with non-invaded biliary and hepatic surgical margins, associated with lymph node dissection seems provides better results compared to biopsies and cholecystectomy alone associated with chemotherapy.

In the event of hepatic or lymph node metastasis, surgical relentlessness allowing the R0 resection of metastasis with appropriate chemotherapy seems to have a benefit on survival. It is noteworthy that our follow-up after the second surgery is not sufficient to evaluate the effectiveness of this aggressive surgical approach, but the survival of more than 26 months observed in our case, seems to be a considerable gain compared to some cases described elsewhere. 


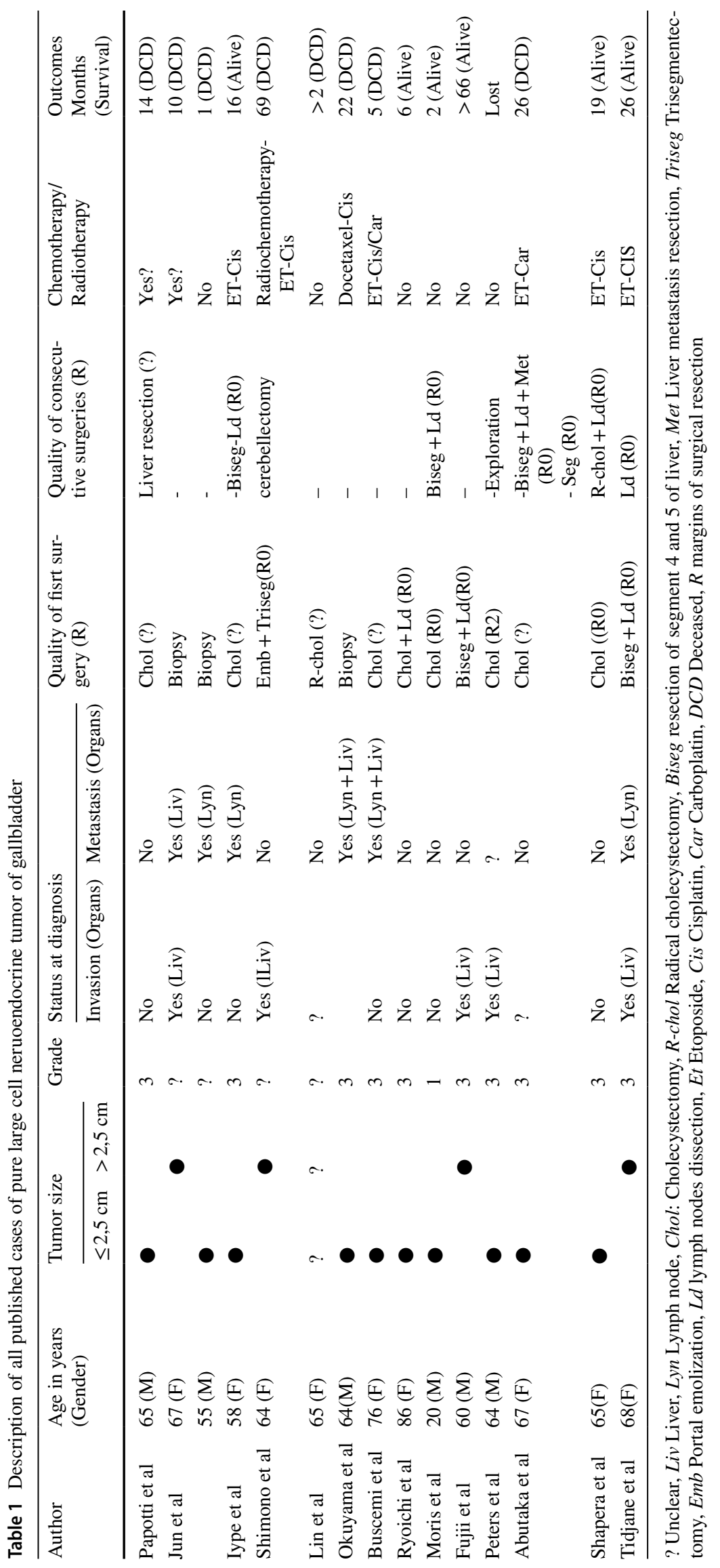




\section{Availability of data and material}

The DATA are not shared, but can be requested directly from the author who will be responsible for transmitting them, after obtaining the patient's agreement.

Acknowledgements To all active members of the West Algerian NET Committee. To Mr. Yassine Terriche for English writing assistance. Dedication: To Pr. Merouane Boukrissa, a hero surgeon, lost on the fields of honor during our battle against the Sars-Cov2 pandemic.

Funding This research did not receive any specific grant from funding agencies in the public, commercial, or not-for-profit sectors.

\section{Compliance with ethical standards}

Conflict of interest All authors declare that they have no competing interests.

Ethics approval Obtained from the ethical council of the EHU-1st November 1954, Oran, Algeria.

Consent for publication Written consent was obtained from the patient for the publication of this article.

\section{References}

1. Choe J, Kim KW, Kim HJ, Kim DW, Kim KP, Hong S-M et al (2019) What Is New in the 2017 world health organization classification and 8th American joint committee on cancer staging system for pancreatic neuroendocrine neoplasms? Korean J Radiol 20(1):5

2. Yao JC, Hassan M, Phan A, Dagohoy C, Leary C, Mares JE et al (2008) One hundred years after 'carcinoid': epidemiology of and prognostic factors for neuroendocrine tumors in 35,825 cases in the United States. J Clin Oncol 26(18):3063-3072

3. Hadoux J, Blanchard P, Scoazec J-Y, Burtin P, Planchard D, Malka $\mathrm{D}$ et al (2019) Post radiation grade 3 neuroendocrine carcinoma (PRNEC): a new entity? Neuroendocrinology 22:120-134

4. Hamdi Cherif M, Bidoli E, Birri S, Mahnane A, Zaidi Z et al (2015) Cancer estimation of incidence and survival in Algeria 2014. J Cancer Res Thera 3(9):100-104

5. Ayabe RI, Wach M, Ruff S, Martin S, Diggs L, Wiemken T et al (2019) Primary gallbladder neuroendocrine tumors: insights into a rare histology using a large national database. Ann Surg Oncol 26(11):3577-3585

6. Papotti M, Cassoni P, Sapino A, Passarino G, Krueger JE, Albores-Saavedra J (2000) Large cell neuroendocrine carcinoma of the gallbladder: report of two cases. Am J Surg Pathol 24(10):1424-1428

7. Haji G, Nabizade U, Kazimov K, Guliyeva N, Isayev I (2019) Liver dose reduction by deep inspiration breath hold technique in right-sided breast irradiation. Radiat Oncol J 37(4):254-258

8. Miyamoto R, Kikuchi K, Uchida A, Ozawa M, Nagai K, Maeda $\mathrm{M}$ et al (2016) Large cell neuroendocrine carcinoma of the gallbladder: a case report and literature review. Int Surg 101(9-10):458-464

9. Jun SR, Lee JM, Han JK, Choi BI (2006) High-grade neuroendocrine carcinomas of the gallbladder and bile duct: report of four cases with pathological correlation. J Comput Assist Tomogr 30(4):604-609

10. Kim T-H, Kim SH, Lee KB, Han JK (2017) Outcome and CT differentiation of gallbladder neuroendocrine tumours from adenocarcinomas. Eur Radiol 27(2):507-517

11. Okuyama Y, Fukui A, Enoki Y, Morishita H, Yoshida N, Fujimoto $S$ (2013) A large cell neuroendocrine carcinoma of the gall bladder: diagnosis with 18FDG-PET/CT-guided biliary cytology and treatment with combined chemotherapy achieved a long-term stable condition. Jpn J Clin Oncol 43(5):571-574

12. Garcia-Carbonero R, Sorbye H, Baudin E, Raymond E, Wiedenmann B, Niederle B et al (2016) ENETS consensus guidelines for high-grade gastroenteropancreatic neuroendocrine tumors and neuroendocrine carcinomas. Neuroendocrinology 103(2):186-194

13. Eilard MS, Lundgren L, Cahlin C, Strandell A, Svanberg T, Sandström P (2017) Surgical treatment for gallbladder cancer - a systematic literature review. Scand J Gastroenterol 52(5):505-514

14. Spada F, Antonuzzo L, Marconcini R, Radice D, Antonuzzo A, Ricci S et al (2016) Oxaliplatin-based chemotherapy in advanced neuroendocrine tumors: clinical outcomes and preliminary correlation with biological factors. Neuroendocrinology 103(6):806-814

15. Auernhammer CJ, Spitzweg C, Angele MK, Boeck S, Grossman A, Nölting $S$ et al (2018) Advanced neuroendocrine tumours of the small intestine and pancreas: clinical developments, controversies, and future strategies. Lancet Diabetes Endocrinol 6(5):404-415

16. Hentic O, Hammel P, Couvelard A, Rebours V, Zappa M, Palazzo $\mathrm{M}$ et al (2012) FOLFIRI regimen: an effective second-line chemotherapy after failure of etoposide-platinum combination in patients with neuroendocrine carcinomas grade 3. Endocr Relat Cancer 19(6):751-757

17. Iype S, Mirza TA, Propper DJ, Bhattacharya S, Feakins RM, Kocher HM (2009) Neuroendocrine tumours of the gallbladder: three cases and a review of the literature. Postgrad Med J 85(1002): 213

18. Abutaka A, El-Matbouly M, Helmy I, Elmoghazy W, Sulieman I, Ben Gashir M et al (2019) Repeat liver resection for pure large cell neuroendocrine carcinoma of the gallbladder: a favorable outcome. World J Surg Oncol 17(1):126

19. Shimono C, Suwa K, Sato M, Shirai S, Yamada K, Nakamura Y et al (2009) Large cell neuroendocrine carcinoma of the gallbladder: long survival achieved by multimodal treatment. Int J Clin Oncol 14(4):351-355

20. Shapera E, Bitting C (2019) Survival : a rare outcome in large cell neuroendocrine carcinoma of the gallbladder. Acta Gastroenterol Belg 82(3):433-436

21. Fujii M, Saito H, Shiode J (2019) Rare case of a gallbladder neuroendocrine carcinoma. Clin J Gastroenterol 12(1):38-45

Publisher's Note Springer Nature remains neutral with regard to jurisdictional claims in published maps and institutional affiliations. 